\title{
Kinetic Study of Bechamp Process for P-Nitrotoluene Reduction to P-Toluidine
}

\author{
Vivek Popat and Nitin Padhiyar
}

\begin{abstract}
Bechamp process is a well-known process for the reduction of aromatic nitro compounds using zero valent iron powder and acid. Reduction of p-nitro toluene (PNT) to p-toluidine (PT) is a process of three steps in series, namely adsorption of PNT on the iron surface, surface reaction of PNT to PT and desorption of the product from the iron surface. Reduction of PNT to PT by Bechamp process has carried out in a $500 \mathrm{ml}$ batch reactor in this work. Gas Chromatograph (GC) is used for the sample analysis. A GC method has been developed with toluene as the solvent for determining the compositions of various reaction components. In this work, we have carried out experiments to find out the limiting step for PNT reduction. We have considered selectivity of PT as performance criteria in this study at various operating conditions. We also present the effect of rpm on rate constant and present the mathematical model for the same.
\end{abstract}

Index Terms-Bechamp process, Frossling correlation, P-nitrotoluene reduction, kinetic study.

\section{INTRODUCTION}

Amine aromatics are important for their applications in pesticides, plasticizers, explosives, inhibitors of metal corrosion, sweetening agents, and as intermediates in pharmaceutical industry [1]. Reduction of aromatic and aliphatic nitro compounds results in their amines counterpart. Often, these aromatic amine components are prepared by reduction of aromatic nitro compounds. Reduction of nitro aromatics has wide application in dye, pharmaceutical and petrochemical industry. The counterpart aromatic amines are used as intermediate for dye and petrochemical products [1], [2]. $\mathrm{NH}_{2}$ group in aromatic amines is one of the chromophore groups (attached group to the benzene ring) in the dye formulation; and $\mathrm{NH}_{2}$ is also the member of auxochrome group which is necessary to impart the solubility and to increase the adherences property [3]. Preferable methods for reductions are catalyst hydrogenation and Bechamp reduction.

Bechamp [2] had invented a process for reducing nitrobenzene to aniline using iron and hydrochloric acid, latter known as Bechamp process. In Bechamp process reduction, aromatic nitro compound is adsorbed on $\mathrm{Fe}^{0}$ surface. Here, it reacts with hydrogen ion $H^{+}$produced from water and acid reaction, which results into production of amine counterpart of aromatic nitro compound. Thus, the presence of solid, gas and liquid phases in the reaction mixture makes this system as three phase system [1]. Bechamp process and the quality of amine product from this

Manscript received July 12, 2013; revised September 11, 2013.

The authors are with the Indian Institute of Technology Gandhinagar (e-mail: nitin@iitgn.ac.in). process are sensitive to the the parameters such as physical state of iron, amount of water used, amount and type of acid used, agitation speed, reaction temperature, and the use of various catalysts or additives [4]. Thus, the quality of the aromatic amine can be controlled by the above mentioned parameters. The Bechamp reduction can be summarized as follow,

Bechamp Reaction:

$H^{+}$Generation:

$$
6 \mathrm{HCOOH}+6 \mathrm{H}_{2} \mathrm{O} \rightarrow 6 \mathrm{HCOO}^{-}+12 \mathrm{H}^{+}+60 \mathrm{H}^{-}
$$

Bechamp reduction reaction:

$$
4 \mathrm{ArNO}_{2}+4 \mathrm{H}_{2} \mathrm{O}+9 \mathrm{Fe}^{0} \rightarrow 4 \mathrm{ArNH}_{2}+3 \mathrm{Fe}_{3} \mathrm{O}_{4}
$$

Bechamp process is the oldest commercial process for preparation of amines, but in more recent years it has been largely replaced by catalytic hydrogenation [5]. Nevertheless, the Bechamp reduction is still used in the dye industry for the production of small volumes of aromatic amines and for the production of iron oxide for pigments [1]. Few problems found in catalytic hydrogenation can be avoided in Bechamp process. For example, catalyst regeneration in catalytic hydrogenation can be avoided in Bechamp process. Further, pharmaceutical grade p-toluidine production, free from catalysts trace is one of the key advantages of Bechamp process over catalytic hydrogenation. Though, due to the slow reaction rate in Bechamp process and costly downstream separation methods, their applications are limited to batch process [6]. For large volumes catalytic reduction finds an edge over the Bechamp process, and vice versa for the small volumes. Small volumes amine production, catalytic regeneration and catalyst losses lead to a costly process compare to Bechamp process. Another application of this process is in the treatment of ground water; because nitro-aromatics present in ground water are not easily biodegradable compare to the amine aromatics. Hence, Bechamp process is used to convert aromatic nitro to aromatic amine in ground water and making them easily biodegradable [7].

Kinetic study for the reduction of nitro benzene using Bechamp process was carried out by Agrawal et al. [7] and Mantha et al. [8]. They focused on the application to the ground water treatment. They proposed and compared different kinetic models for the NB conversion to aniline. In their work, they found that mass transfer is the limiting step for nitrobenzene reduction [7]. Effect of various parameters affecting kinetics such as $\mathrm{pH}$, iron surface area and agitation speed were studied by Agrawal et al. [7], Mu et al. [9] and Choe et al [10]. Though, similar work for the reduction of 
p-nitro-toluene using Bechamp process has not been found in the open literature to the best of authors' knowledge. This work focuses on Bechamp reduction of p-nitrotoluene to p-toluidine. We have carried out experimental study of the Bechamp process for p-nitro toluene in a lab scale batch reactor with two reaction parameters, namely reactor temperature and agitation speed. A method in Gas Chromatography (GC) has been developed for finding the compositions of $\mathrm{p}$-nitro toluene and $\mathrm{p}$-toluidine.

\section{REACTION MECHANISM}

Reduction mechanism for aromatic nitro compounds can be explained by considering reduction mechanism as a three step process, namely (1) generation of $\mathrm{H}^{+}$ions by water-acid reaction, (2) reaction of $\mathrm{H}^{+}$with p-nitrotoluene, and (3) oxidation of $\mathrm{Fe}^{0}$ by $\mathrm{H}^{+}$. These reactions [7] are explained in the following section,

\section{A. $\mathrm{H}^{+}$Generation}

First step in the Bechamp process is the production of hydrogen ion, which is produced by reaction between acid and water. Formic acid and hydrochloric acid are commonly used acids in Bechamp process. Since, halogen group in hydrochloric acid is quite reactive and hence may react with the aromatic ring; formic acid is more popular than $\mathrm{HCl}$.

Acid - water Reaction:

$$
\begin{gathered}
6 \mathrm{HCOOH}+6 \mathrm{H}_{2} \mathrm{O} \rightarrow 6 \mathrm{HCOO}^{-}+6 \mathrm{OH}^{-}+12 \mathrm{H}^{+} \\
6 \mathrm{H}^{+}+6 \mathrm{HCOO}^{-} \rightarrow 6 \mathrm{HCOOH} \\
\text { Overall reaction: } 6 \mathrm{H}_{2} \mathrm{O} \rightarrow 12 \mathrm{H}^{+}+6 \mathrm{OH}^{-}
\end{gathered}
$$

Overall reaction results in only water decomposition and acid does not get consumed. Hence, quite small amount of acid can be sufficient for the requirement of hydrogen ion in Bechamp process. It is reported in the literature that amine is not detected in the strong acidic conditions [7]. Further, it is also suggests that 5 to 6.9 is the optimum $\mathrm{pH}$ in the Bechamp process.

\section{B. Reduction of P-Nitrotoluene}

Reduction of p-nitrotoluene can be summarized as a three step process, adsorption of PNT from bulk phase to iron surface, reaction of the same on iron surface, and desorption of the reaction products from iron surface to the bulk phase. Adsorption of p-nitrotoluene on the Fe $\mathrm{O}^{0}$ surface:

$$
\begin{aligned}
& \mathrm{P} \text {-nitrotoluene is adsorbed on zero valent iron } \rightarrow \mathrm{ArNO}_{2}+ \\
& \mathrm{Fe}^{0} \\
& \text { C. Reaction between P-Nitrotoluene and Hydrogen Ion on } \\
& \text { Fe Surface }
\end{aligned}
$$

\section{Reaction between P-Nitrotoluene and Hydrogen Ion on Fe Surface}

This is a complex reaction, a result of series and parallel reactions, with generation of one or more intermediates and byproducts.

$$
\mathrm{ArNO}_{2}+\mathrm{Fe}^{0}+\mathrm{H}^{+} \rightarrow \mathrm{ArNH}_{2}+\mathrm{Fe}^{2+}
$$

Literature suggests that reaction of aromatic nitro to its amine counterpart is non-elementary reaction, with two or three byproducts generation depending upon the reaction conditions. Many authors have proposed reaction mechanism for nitro aromatic compounds and especially for nitro benzene. Firstly, p-nitrotoluene reduces to p-nitrosotoluene followed by $\mathrm{p}$-nitrosotoluene reduction to p-phenylhydroxytoluene, and finally the reduction of p-phenylhydroxytoluene to p-toluidine. Similar to the reduction of nitro benzene [7], the reduction of p-nitrotoluene can be summarized in three steps as follows,

$$
\begin{aligned}
& \mathrm{ArNO}_{2}+\mathrm{Fe}^{0}+2 \mathrm{H}^{+} \rightarrow \operatorname{ArNO}+\mathrm{Fe}^{2+}+\mathrm{H}_{2} \mathrm{O} \\
& \mathrm{ArNO}+\mathrm{Fe}^{0}+2 \mathrm{H}^{+} \rightarrow \operatorname{ArNHOH}+\mathrm{Fe}^{2+} \\
& \mathrm{ArNHOH}+\mathrm{Fe}^{0}+2 \mathrm{H}^{+} \rightarrow \mathrm{ArNH}_{2}+\mathrm{Fe}^{2+}+\mathrm{H}_{2} \mathrm{O}
\end{aligned}
$$

First reaction corresponds to two electron reduction of p-nitrotoluene to p-nitrosotoluene; the second one corresponds to four electron reduction of $\mathrm{p}$-nitrosotoluene to phenylhydroxytoluene and third corresponds to two electron reduction of phenylhydroxytoluene to p-toluidine. Note that these three reactions are the series reactions only [7].

\section{Oxidation of $\mathrm{Fe}^{0}$}

Above mentioned all the three reactions result in the oxidized state of $\mathrm{Fe}$, i.e. $\mathrm{Fe}^{2+}$. This in turn reacts with $\mathrm{OH}^{-}$ion that was produced from water dissociation and produces ferrous hydroxide, $\mathrm{Fe}(\mathrm{OH})_{2}$. This $\mathrm{Fe}(\mathrm{OH})_{2}$ can still provide free surface for adsorption of p-nitrobenzene, that is similar to zero-valent iron surface. Further, on the surface of ferrous hydroxide, p-nitrobenzene reduces to the amine counterpart with further oxidation of $\mathrm{Fe}(\mathrm{OH})_{2}$ to $\mathrm{Fe}(\mathrm{OH})_{3}$. This can be summarized as follows [7], [1],

$$
\begin{gathered}
3 \mathrm{Fe}^{2+}+6 \mathrm{OH}^{-} \rightarrow 3 \mathrm{Fe}(\mathrm{OH})_{2} \\
\mathrm{ArNO}_{2}+6 \mathrm{Fe}(\mathrm{OH})_{2}+4 \mathrm{H}_{2} \mathrm{O} \rightarrow 6 \mathrm{Fe}(\mathrm{OH})_{3}+\mathrm{ArNH}_{2} \\
\mathrm{Fe}(\mathrm{OH})_{2}+2 \mathrm{Fe}(\mathrm{OH})_{3} \rightarrow \mathrm{Fe}_{3} \mathrm{O}_{4}+4 \mathrm{H}_{2} \mathrm{O}
\end{gathered}
$$

Though, the above mechanism results into only two $\mathrm{Fe}$ compounds, namely $\mathrm{Fe}(\mathrm{OH})_{2}$ and $\mathrm{Fe}_{3} \mathrm{O}_{4}$, a mixture of different iron-oxide reactions are reported in the literature [1]. Composition of iron-oxide depends on the reaction conditions, though not significant experimental results have been demonstrated to support this claim.

An overall reaction including nitro reduction and $\mathrm{Fe}$ oxides generation can be shown below,

$$
4 \mathrm{H}_{2} \mathrm{O}+4 \mathrm{ArNO}_{2}+9 \mathrm{Fe}^{0} \rightarrow 4 \mathrm{ArNH}_{2}+3 \mathrm{Fe}_{3} \mathrm{O}_{4}
$$

\section{Chemical And Experimental SET-UP}

Para-nitrotoluene with $99.9 \%$ purities in a form of yellow crystal (N27322) and Para-toluidine with $99.7 \%$ purity in the form of white pellet (Aldrich -236314) were purchased from sigma-Aldrich. Iron powder with low magnesium and manganese content, with purities of $99.5 \%$ of a size range 6 $9 \mu \mathrm{m}$ was purchased from sigma-Aldrich. Toluene of HPLC and/or GC grade was used as a solvent for Gas chromatography analysis. Water used in the reaction is RO 
treated water, which is free from dissolved solid and other impurities. The experimental setup consists of a five- neck conical flask reactor, electrical heater, agitator, and a reflux condenser.

\section{MAThematic Model}

Reduction of p-nitrotoluene (PNT) by Bechamp process under wide operating conditions results into p-toluidine (PT) with/without intermediate/byproducts. Our GC results of liquid phase compositions show only two peaks, of PNT and PT, with RTD of $3.53 \mathrm{~min}$ and $2.90 \mathrm{~min}$, respectively. Hence, we have ignored the formation of intermediates and byproducts for modeling purpose. The composition of aromatic compounds in the liquid phase was found by GC with an assumption that there is no accumulation of these compounds on the iron surface.

It is believed that the conversion from PNT to PT is a result of the following three steps in series: (1) adsorption of PNT on the iron surface followed by, (2) reaction on iron surface, and (3) desorption of the product [11].

Agrawal et al. [7] suggested that adsorption is the limiting step for the reduction of aromatic nitro compounds with an example of nitrobenzene reduction. Firstly, we make a hypothesis of adsorption as the limiting step for PNT to PT reduction. Next, we present experimental results to verify the hypothesis.

With adsorption as the rate limiting step,

$$
-r_{A}=k_{m}\left(C_{A b}-C_{A s}\right)
$$

where, $-r_{A}$ is the rate of adsorption of $A$ (PNT), $k_{m}$ is mass transfer constant, $C_{A b}$ is the concentration of $A$ in Bulk liquid, and $C_{A s}$ is the concentration of $A$ on the iron surface. According to the hypothesis of adsorption being the slowest step, $C_{A s}$ can be assumed to be very small compare to $C_{A b}$. Thus, ignoring $C_{A s}$ in "(15)".

$$
-r_{A}=k_{m}\left(C_{A b}-0\right)
$$

Hence

$$
\frac{d C_{A}}{d t}=k_{m} * C_{A b}
$$

Or upon integration,

$$
\ln \left(\frac{C_{A 0}}{C_{A}}\right)=k_{m} * t
$$

where, $C_{A 0}$ is the initial concentration in the bulk phase. Thus, if adsorption is the limiting step, equations "(17)" or "(18)" should be satisfied. Please note that a first order of reaction with reaction being the limiting step may also satisfy the above equation "(17)". Hence, to confirm the mass transfer being the limiting step, we conducted experiments at different rpm in the range of 320-680. Further, Frossling correlation [11] of rpm and the rate constant shown in "(19)" can be fitted in the case of mass transfer as the limiting step.

$$
S h=2+0.6 R e^{1 / 2} S c^{1 / 3}
$$

where, $S h$ is the Sherwood number $\left(k d_{p} / D_{A B}\right), S c$ is Schmidt number $\left(v / D_{A B}\right), \mathrm{k}$ is the mass transfer constant, $\mathrm{dp}$ is the iron particle diameter, $D_{A B}$ is the Diffusivity coefficient, $V$ is angular velocity, and $v=$ kinematic velocity. As can be observed from the above Frossling equation, rate constant is proportional to the square root of rpm at constant temperature and particle diameter.

$$
k \propto(r p m)^{1 / 2}
$$

\section{RESUlt AND DiscUSSION}

\section{Reduction Kinetics}

To see the effect of rpm on kinetics of PNT reduction, we carry out experiments at various rpm in the range of 320-680. All the experiments were performed with PNT at an initial composition of $1 \mathrm{gm}$ in $300 \mathrm{ml}$ of water; reaction Temperature of $100{ }^{\circ} \mathrm{C}$ and 5-5.5 $\mathrm{pH}$. The transient batch reactor data in terms of PNT and PT concentrations at 500 rpm are shown in Fig. 1

The reaction kinetics shows that the reaction time for $98 \%$ conversion of PNT is $20 \mathrm{~min}$. For The kinetic constant, $k$, from the slope of $\ln \left(C_{A O} / C_{A}\right)$ Vs $t$ was found to be $0.244 \mathrm{~min}^{-1}$ with the $R^{2}$ value of 0.989 as shown Figs. 2-3 summarizes the effect of rpm on the overall rate of reduction of PNT at four different rpm values, 320, 500, and 680. As can be observed from Fig. 3, the rate of conversion of PNT increases with an increase in rpm from 320 to 680 . This indicates that the adsorption is the rate limiting step.

To obtain $98 \%$ conversion, it took 60 min of time with 320 rpm, while it was 20 min and 15 min with 500 and $680 \mathrm{rpm}$. Thus, increase in the agitation speed enhanced the supply rate of the reactant on the solid surface for the reaction to occur.

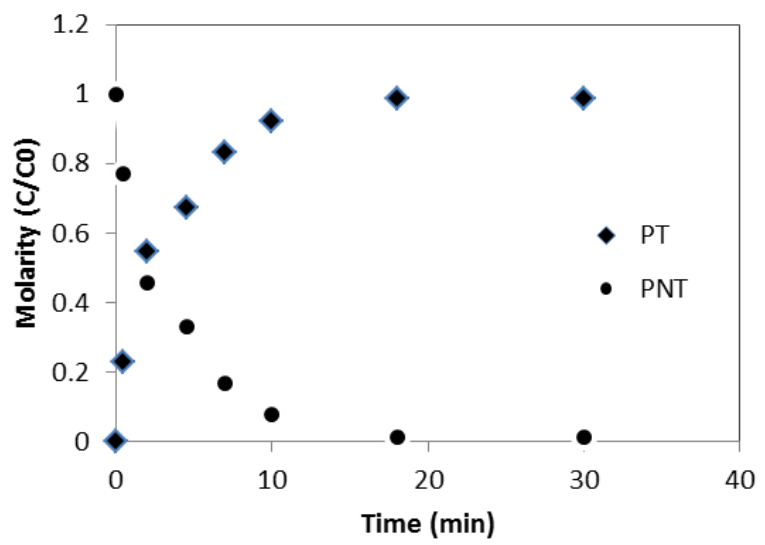

Fig. 1. Kinetics of PNT reduction with an initial composition of $1 \mathrm{gm}$ of PNT in $300 \mathrm{ml}$ of water with $1 \mathrm{gm}$ of iron addition

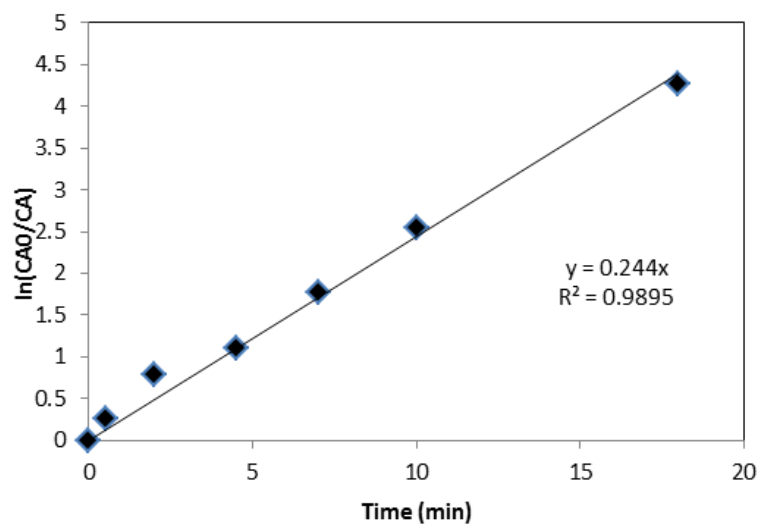

Fig. 2. Temporal plot of composition of PNT for the first order kinetics 


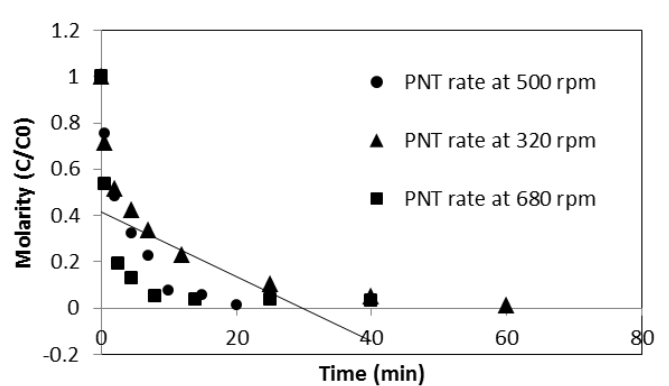

Fig. 3. Effect of rpm on the reduction rate of PNT for rpm range 320 to 680.

Please note that the effect of rpm on the kinetic constant should be of the nature as shown in Fig. 4 [11]. At low rpm, the boundary layer thickness is large and adsorption limits the reduction reaction. Through increase in $\mathrm{rpm}$, reduces the boundary layer thickness and at some high rpm the adsorption no longer remains as the limiting step as shown in Fig. 4 [11].

We found the relation of $k$ vs rpm following the trend shown in Fig. 5 for the rpm range of 320-680. Though, at a very high rpm of 1000 , a reverse trend was observed, which is counter intuitive as shown in Fig. 5. Kinetic data of PNT at two rpm values, 680 and 1000 are shown in Fig. 5. As can be observed, reaction is faster with $680 \mathrm{rpm}$ compare to 1000 rpm, which is counter intuitive. Though, at such a high rpm, without baffles in the vessel, centrifugal force became more dominating than mixing of reaction mass and the reaction mixture moves similar to a rigid body hindering the proper mixing [7],[12]. This adversely affectS the mass transfer, which in turn retards the reduction rate of PNT.

Thus, the graph of $k V s \sqrt{r p m}$ of should be straight line. We conducted experiments at three different rpm values, 320, 500 and 680 and found the rate constants $0.077,0.244$ and 0.339 . The least square fit of the above model for these data is shown in Fig. 6. As discussed in the previous section, for mass transfer being the limiting step, the rate constant, $k$ should be proportional to the square root of rpm. The plot of $k$ $\mathrm{Vs} \sqrt{r p m}$ is shown in Fig. 6 with $\mathrm{R}^{2}$ fit of 0.998 . Thus, a satisfactory fit of the Frossling model was obtained to represent the experimental data. This suggests that the reduction of PNT to PT is a mass transfer controlled reaction.

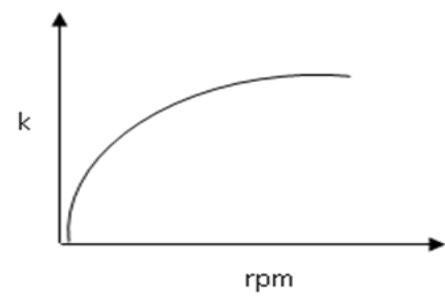

Fig. 4. Effect of rpm on kinetic constant a general approach [11]

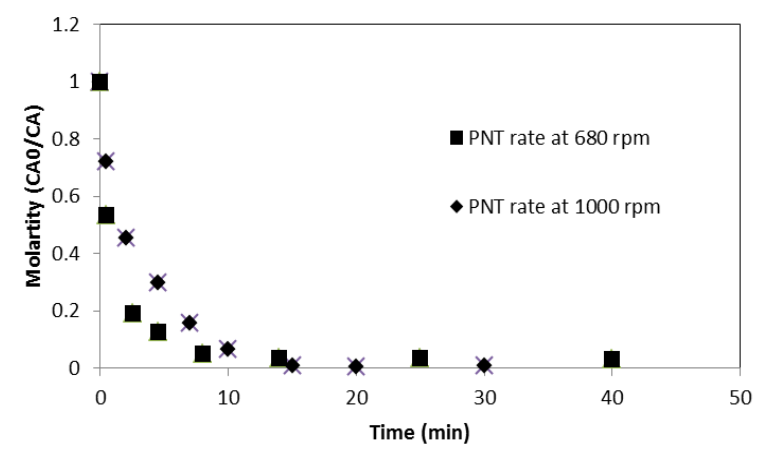

Fig. 5. Effect of rpm on the reduction rate of PNT for rpm range 680 to 1000 .

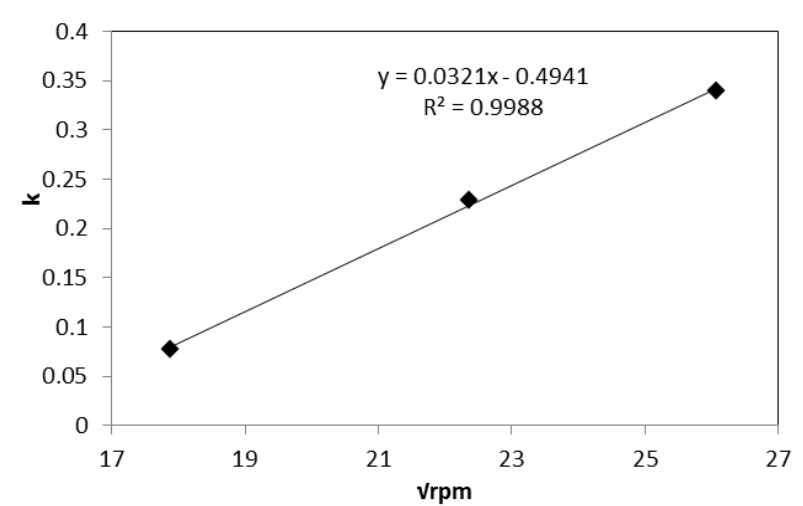

Fig. 6. Kinetic constant, $k$ as a function of $\sqrt{r p m}$ for rpm range of 320 to 680 .

\section{CONCLUSION}

Bechamp reduction is a reaction carried out on the surface of iron in acidic medium. Reduction of all nitro-aromatics to amine-aromatics follows series reaction with the two intermediates namely nitroso-aromatics and phynlhydroxy-aromatics. PNT reduces to PT with different by products depending upon the reaction condition. In PNT reduction; we had found higher selectivity of PT or higher production of PT compare to negligible production of other side products.

The overall conversion of PNT to PT is best described by three steps in series, adsorption of PNT on iron surface, reaction of PNT to PT on $F e$ surface, and desorption of PT to the bulk liquid phase. Among that Adsorption of PNT from the bulk liquid to the iron surface was found to be the controlling step of the overall reduction rate in the operating conditions considered in this work. At fairly high temperature of $100{ }^{\circ} \mathrm{C}$, rate of desorption and rate of reaction are higher than that of the adsorption rate. Higher temperature is required to facilitate effective desorption of amine from the surface of the iron. Experimental results show that reduction of PNT to PT was a mass transfer control with the rate constant value of $0.077 \mathrm{~min}^{-1}$ at $320 \mathrm{rpm}, 0.244 \mathrm{~min}^{-1}$ at 500 rpm and $0.339 \mathrm{~min}^{-1}$ at $680 \mathrm{rpm}$. In all the three cases, the model with adsorption limiting step, was satisfactorily fitting the experimental data.

The effect of rpm on the rate constant was also satisfactorily verified by Frossling correlation. As per the Frossling correlation, $k$ should be proportional to $\sqrt{r p m}$ which was confirmed by the experimental data in this work for PNT reduction. Though, the experimental results at higher rpm ( 1000), was not found to fit the Frossling correlation. In fact, at $1000 \mathrm{rpm}$, the rate of conversion of PNT decreased compare to that at lower rpm, which is counter intuitive. This trend can be attributed to the fact that at high rpm centrifugal force is dominating.

\section{REFERENCES}

[1] K. Othmer, “Amine by Reduction,” Enyclopindia, vol. 2, pp. 476-490, 2004.

[2] Bechamp, "Bechamp reduction," Ann. J. Am. Chem. Phy., vol. 42, pp. $186,1854$.

[3] C. E. Dryden, "Outlines of chemical technology," East-West Press, 1973.

[4] K. T. Hindle, S. D. Jackson, D. Stirling, and G. webb, "The hydrogenation of para-toluidine over rhodium/silica; the effect of metal particle size and support texture," J. Catalys., vol. 241, pp. 417-425, 2006. 
[5] N. Ono, "The nitro Group in Organic Synthesis," Wiley 2001, pp. $172-175,2001$.

[6] A. Agrawal and P. G.Tratnyek, "Reduction of Nitro Aromatic Compounds by Zero-valent Iron Metal," J. Env. Sci. Tech., vol. 30, pp. 153-160, 1996.

[7] Z. Wang, "Comprehensive Organic Name Reactions and Reagents," Wiley, pp. 284-287, 2010.

[8] A. Agrawal and P. G. Tratnyek, "Reduction of Nitro Aromatic Compounds by Zero-valent Iron Metal," J. Env. Sci. Tech., vol. 30, pp. 153-160, 1996.

[9] R. Mantha, K. E. Taylor, N. Biswas, and J. K. Bewtra, "A Continuous system for $\mathrm{Fe}^{0}$ reduction of nitrobenzene in synthetic wastewater," $J$. Env. Sci. Tech., vol. 35, no. 15, pp. 3231-3236, 2001.

[10] Y. Mu, H. Yu, J. Zheng, S. zhang, and G. Sheng, "Reduction degradation of nitrobenzene in aqueous solution by zero-valent iron," Journal of Chemosphere, vol. 54, pp. 789-794, 2004

[11] S. Choe, H. M. Liljestrand, and J. Khim, "Nitrate reduction by zero-valent iron under different $\mathrm{pH}$ regimes," J. Applied Geochem., vol. 19, pp. 335-342, 2004

[12] H. S. Fogler, "Eleements of Chemical Reaction Engineering," pp. 253-310 and 757-810, 2011.

[13] W. L. Cabe, J. C. Smith, and P. Harriott, Unit Operations of Chemical Engineering, $7^{\text {th }}$ Edition, McGraw-Hill International Edition, pp. $249-251,2005$

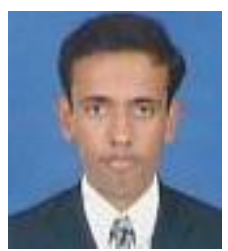

Vivek Popat was born on $16^{\text {th }}$ Aug 1990, in Rajkot. $\mathrm{He}$ is a post graduate student of Indian Institute of Technology, Gandhinagar, India. Mr. Popat presented his work on semibatch reactor for kinetic study of nitroatomatic compounds in Chemference' 12 at IIT Bombay in December 2012.

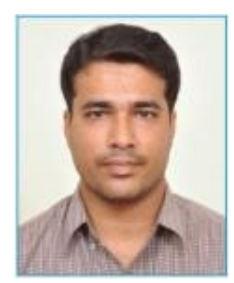

Nitin Padhiyar was born on 27 Dec, 1978, in Jamnagar, India. He is working as an ASSISTANT PROFESSOR in Chemical Engineering at IIT Gandhinagar. $\mathrm{He}$ worked for one year at $\mathrm{ABB}$ Bangalore in 2007-2008. His current research area focuses on process control and optimization and reaction kinetics. Dr. Padhiyar received Ambuja Young Researcher;s award for doing Ph.D. in India based on the GATE score in 2004. He received Indo-US research Fellowship for doing research for one year at CMU, Pittsburgh, USA in 2009-10. 